\title{
Prevalência e fatores associados à hipertensão em idosos de um serviço de atenção primária
}

\section{Prevalence and factors associated with hypertension in elderly people at a primary care service}

\section{Prevalencia y factores asociados a la hipertensión en ancianos de un servicio de atención primaria}

Recebido: $24 / 07 / 2017$

Aprovado: 18/10/2017

Publicado: 07/05/2018

\section{Gerson Souza Santos ${ }^{1}$ Isabel Cristina Kowal Olm Cunha ${ }^{2}$}

A hipertensão arterial sistêmica é uma doença altamente prevalente em indivíduos idosos, tornando-se um fator determinante na morbidade e mortalidade elevadas dessa população. 0 objetivo deste estudo é analisar as características epidemiológicas da hipertensão arterial sistêmica (HAS) e os fatores associados na população idosa acompanhada por uma Unidade Básica de Saúde da zona Sul da cidade de São Paulo. Trata-se de um estudo transversal realizado por meio de inquérito domiciliar com 340 idosos. Os idosos que participaram deste estudo eram sua maioria mulheres (62\%), na faixa etária de 60 a 69 anos A prevalência da hipertensão foi de 74,7\%. Os fatores associados à hipertensão foram: sexo masculino (p-valor 0,0133 ) e raça negra (p-valor 0,0365). A HAS apresentou alta prevalência nos idosos investigados, sendo maior em determinados subgrupos: homens, baixa escolaridade e raça negra. 0 aumento da população idosa, evidencia maior número de problemas crônicos, entre elas a hipertensão arterial.

Descritores: Hipertensão; idoso; Doença crônica; Epidemiologia.

Arterial hypertension is a systemic disease highly prevalent in elderly individuals and a decisive factor in high morbidity and mortality in this population. The aim of this study is to assess the epidemiological characteristics of hypertension (SAH) and the associated factors in elderly population accompanied at a Basic Health Unit in the southern region of São Paulocity. This is a cross-sectional study carried out through investigation at home with 340 elderlies. The elderly who participated in this study were mostly women (62\%) from 60 to 69 years old. The prevalence of hypertension was 74.7\%. The factors associated with hypertension were male gender ( $\mathrm{p}$-value 0.0133 ) and black-skinned ( $p$-value 0.0365). HAS presented high prevalence in the elderly investigated, being higher in certain subgroups: men, low education level and black race. The increase of elderly population shows greater number of chronic problems, including high blood pressure.

Descriptors: Hypertension; Aged; Chronic disease; Epidemiology.

La hipertensión arterial sistémica es una enfermedad altamente prevalente en individuos ancianos, volviéndose un factor determinante en la morbilidad y mortalidad elevadas de esa población. El objetivo de este estudio es analizar las características epidemiológicas de la hipertensión arterial sistémica (HAS) y los factores asociados en la población anciana acompañada por una Unidad Básica de Salud de la zona sur de la ciudad de São Paulo. Se trata de un estudio transversal realizado por medio de una encuesta domiciliaria con 340 ancianos. Los participantes eran en su mayoría mujeres (62\%), en el grupo de edad de 60 a 69 años. La prevalencia de la hipertensión fue de 74,7\%. Los factores asociados a la hipertensión fueron: sexo masculino ( $p$-valor 0,0133 ) y raza negra ( $p$ valor 0,0365 ). La HAS presentó alta prevalencia en los ancianos investigados, siendo mayor en determinados subgrupos: hombres, baja escolaridad y raza negra. El aumento de la población anciana, evidencia mayor número de problemas crónicos, entre ellos la hipertensión arterial.

Descriptores: Hipertensión; Anciano; Enfermedad crónica; Epidemiología.

1. Enfermeiro. Mestre em Enfermagem. Doutor em Ciências. Enfermeiro Sanitarista na Estratégia Saúde da Família pela Associação Paulista para o Desenvolvimento da Medicina, São Paulo, SP, Brasil. ORCID: 0000-0002-6084-7313 E-mail: enf.gerson@hotmail.com

2. Enfermeira. Mestre em Enfermagem. Doutora em Saúde Pública. Livre Docente em Administração em Enfermagem. Professora Associada aposentada da Universidade Federal de São Paulo, São Paulo, SP, Brasil. ORCID: 0000-0001-6374-5665 E-mail: Isabelcunha@hotmail.com 


\section{INTRODUÇÃO}

envelhecimento populacional é um

$\mathrm{O}$ fenômeno mundial que se configura como um dos eventos mais significativos da sociedade, adquirindo, ao longo dos anos, dimensões mais expressivas, particularmente nos países em desenvolvimento ${ }^{1}$. No Brasil, o segmento populacional representado pelos idosos é o que mais cresce. Projeções apontam que em 2025 o país ocupará o sexto lugar entre aqueles com maior número de idosos, quando aproximadamente $15 \%$ dos brasileiros terão idade igual ou superior a 60 anos, o que representa, em valores absolutos, 32 milhões de pessoas ${ }^{2}$.

Se por um lado o envelhecimento populacional trouxe os benefícios de uma maior longevidade, por outro aumentou a ocorrência do perfil de morbimortalidade, caracterizado por um aumento de doenças crônico-degenerativas ${ }^{3}$. A preocupação com as condições de saúde do idoso tem motivado o desenvolvimento de vários estudos sobre o envelhecimento humano. Essas pesquisas são essenciais no direcionamento de políticas públicas que atendam à parcela idosa da população, mesmo porque o atual sistema de saúde brasileiro ainda precisa ser ajustado e organizado para os diferentes perfis demográficos e epidemiológicos decorrentes do aumento da expectativa de vida ${ }^{3}$.

Com o aumento de idosos, é significativa a prevalência de doenças crônicas, as maiores causas de morbidade e mortalidade no mundo. Entre essas doenças destacam-se a Hipertensão Arterial Sistêmica (HAS) a Diabetes Mellitus (DM) e o Acidente VascularEncefálico (AVE). No Brasil, as doenças cardiovasculares representam a principal causa de mortalidade, revelando dificuldades em seu controle, sobretudo quando são assintomáticas, como é o caso da HAS $^{4}$.

A HAS é considerada uma doença e um fator de risco, diretamente relacionada a doença arterial coronariana e acidente vascular encefálico, representando um grande desafio para a saúde pública, pois as doenças cardiovasculares constituem a primeira causa de morte no Brasil. A detecção, o tratamento e o controle da HAS são fundamentais para a redução dos eventos cardiovasculares 5 .

0 crescimento acelerado da população de idosos e os aspectos a ela inerentes e, o fenômeno do envelhecimento constitui uma questão atual, o que se faz necessário conhecer o perfil epidemiológico, as características e os fatores associados à HAS nessa população. Acredita-se ser possível a prevenção de doenças crônicas e suas sequelas, controle de suas causas e fatores de risco, com o incremento de ações e programas de prevenção $0^{6,7}$.

0 objetivo deste estudo foi analisar as características epidemiológicas da hipertensão arterial sistêmica (HAS) e os fatores associados na população idosa acompanhada por uma Unidade Básica de Saúde.

\section{MÉTODO}

Trata-se de um estudo de delineamento transversal, de base populacional, realizado por meio de inquérito domiciliar. Com 340 idosos de idade igual ou superior a 60 anos, de ambos os sexos, residentes em uma área de abrangência da Unidade Básica de Saúde (UBS) Vila Santa Catarina, localizada na zona sul do município de São Paulo-SP.

A cidade de São Paulo apresenta um rápido processo de envelhecimento populacional em plena transição demográfica. Concomitantemente, está vivenciando o processo de transição epidemiológica, com aumento da prevalência de condições crônicas e mudança do perfil de morbimortalidade de sua população. Atualmente, São Paulo tem 11.638.802 habitantes, sendo 1.619 .760 de pessoas com 60 anos e mais ${ }^{8}$. Para este estudo adotou-se a técnica de amostragem aleatória simples sem reposição.

A coleta de dados foi realizada pelos pesquisadores por meio de formulários testados, no período de janeiro a março de 2013. Foram obtidas informações sobre identificação do indivíduo, variáveis demográficas, socioeconômicas, estilo de vida, morbidade referida, polifarmácia, incapacidade para Atividades Básicas da vida diária (ABVD), incapacidade para atividades 
instrumentais da vida diária (ABVD) e hospitalização. As variáveis demográficas e socioeconômicas estudadas foram: sexo, idade, cor da pele, situação conjugal, escolaridade e renda familiar.

As categorias para o tabagismo foram: fumantes, não fumantes e ex-fumantes. 0 consumo de bebidas alcoólicas foi categorizado em "sim" e "não", questionados em relação aos 30 dias anteriores à entrevista. A atividade física no lazer foi categorizada em "sim" e "não". A variável polifarmácia foi considerada quando o idoso utilizava 5 ou mais medicamentos de diferentes classes farmacológicas ${ }^{9}$.

Para este estudo foram estabelecidos os seguintes critérios de inclusão: ter 60 anos ou mais; estar cadastrado na Unidade Básica de Saúde e concordar em participar do estudo através da assinatura do Termo de consentimento Livre e Esclarecido (TCLE).

Em relação ao conhecimento da condição de hipertensão, foram considerados os indivíduos que autoreferiram saber desta condição. Quanto ao tratamento foram considerados os idosos que referiram o uso de anti-hipertensivos.

Para a análise estatística foi utilizado o software Statistical Analysis System 9.9 (SAS), que permite a análise da consistência dos dados. Para a verificar a existência entre a variável dependente HAS e demais variáveis independentes do estudo, foi utilizada a técnica de regressão logística, teste quiquadrado e a medida de OddsRatio (OR).

0 projeto de pesquisa foi avaliado pelo Comitê de Ética em Pesquisa da Universidade Federal de São Paulo № 1012/11 e do Comitê de Ética em Pesquisa da Secretaria Municipal de Saúde do município de São Paulo CAAE: 0813.0.174.162.11.

\section{RESULTADOS}

A média de idade dos participantes foi de 68,9 anos com desvio padrão de 7,3 anos. Idade mínima 60 anos e máxima de 85 anos, $62,1 \%$ eram amulheres na faixa etária de 60 a 69 anos, pardos e negros 58\%, baixa escolaridade constituída de 1 a 4 anos de estudo, com renda familiar de 1 a 3 salários mínimos, $86 \%$ eram sedentários com incapacidade para o desempenho das atividades instrumentais da vida diária $86 \%$, como na Tabela 1.

A prevalência de HAS em idosos foi de $74,7 \%$, as variáveis sexo e raça apresentaram significância estatística, indicando que Idosos do sexo masculino apresentaram duas vezes mais chances de hipertensão do que o sexo feminino, assim como os idosos de raça negra apresentaram duas vezes mais chances de hipertensão do que os de raça parda (Tabela 2). 
Tabela 1. Prevalência de hipertensão arterial, segundo variáveis demográficas, socioeconômicas, estilo de vida e capacidade funcional de idosos usuários da UBS Vila Santa Catarina, município de São Paulo-SP, 2013.

\begin{tabular}{|c|c|c|c|c|c|c|c|}
\hline & \multirow{2}{*}{ Variáveis } & \multicolumn{2}{|c|}{ Sim } & \multicolumn{2}{|c|}{ Não } & \multirow{2}{*}{ Total } & \multirow{2}{*}{ p-valor } \\
\hline & & $\mathrm{N}$ & $\%$ & $\mathrm{~N}$ & $\%$ & & \\
\hline \multirow{6}{*}{ Idade } & 60 a 64 anos & 86 & 77.5 & 25 & 22.5 & 111 & \multirow{6}{*}{0.6657} \\
\hline & 65 a 69 anos & 71 & 71.7 & 28 & 28.3 & 99 & \\
\hline & 70 a 74 anos & 30 & 68.2 & 14 & 31.8 & 44 & \\
\hline & 75 a 79 anos & 36 & 78.3 & 10 & 21.7 & 46 & \\
\hline & 80 a 85 anos & 31 & 77.5 & 9 & 22.5 & 40 & \\
\hline & Total & 254 & & 86 & & 340 & \\
\hline \multirow{3}{*}{ Sexo } & Masculino & 106 & 82.2 & 23 & 17.8 & 129 & \multirow{3}{*}{0,0133} \\
\hline & Feminino & 148 & 70.1 & 63 & 29.9 & 211 & \\
\hline & Total & 254 & & 86 & & 340 & \\
\hline \multirow{4}{*}{ Raça/Cor } & Branca & 74 & 68.5 & 34 & 31.5 & 108 & \multirow{4}{*}{0,0365} \\
\hline & Negra & 75 & 84.3 & 14 & 15.7 & 89 & \\
\hline & Parda & 105 & 73.4 & 38 & 26.6 & 143 & \\
\hline & Total & 254 & & 86 & & 340 & \\
\hline \multirow{5}{*}{ Estado civil } & Solteiro & 66 & 79.5 & 17 & 20.5 & 83 & \multirow{5}{*}{0.5445} \\
\hline & Casado & 74 & 71.8 & 29 & 28.2 & 103 & \\
\hline & Divorciado & 27 & 69.2 & 12 & 30.8 & 39 & \\
\hline & Viúvo & 87 & 75.7 & 28 & 24.4 & 115 & \\
\hline & Total & 254 & & 86 & & 340 & \\
\hline \multirow{3}{*}{ Escolaridade } & Analfabeto & 126 & 77.3 & 37 & 22.7 & 163 & \multirow{3}{*}{0.2909} \\
\hline & $\begin{array}{l}\text { Ensino fundamental } \\
\text { incompleto }\end{array}$ & 128 & 72.3 & 49 & 27.7 & 177 & \\
\hline & Total & 254 & & 86 & & 340 & \\
\hline \multirow{3}{*}{ Renda familiar } & 1 a 3 salários mínimos & 197 & 74.6 & 67 & 25.4 & 264 & \multirow{3}{*}{0.9466} \\
\hline & 4 a 5 salários mínimos & 57 & 75.0 & 19 & 25.0 & 76 & \\
\hline & Total & 254 & & 86 & & 340 & \\
\hline \multirow{3}{*}{$\begin{array}{l}\text { Internação } \\
\text { hospitalar }\end{array}$} & Sim & 88 & 73.3 & 32 & 26.7 & 120 & \multirow{3}{*}{0.6672} \\
\hline & Não & 166 & 75.5 & 54 & 24.6 & 220 & \\
\hline & Total & 254 & & 86 & & 340 & \\
\hline \multirow{3}{*}{$\begin{array}{l}\text { Atividade } \\
\text { física }\end{array}$} & Não & 205 & 75.1 & 68 & 24.9 & 273 & \multirow{3}{*}{0.7412} \\
\hline & Sim & 49 & 73.1 & 18 & 26.9 & 67 & \\
\hline & Total & 254 & & 86 & & 340 & \\
\hline & Fumante & 48 & 73.9 & 17 & 26.2 & 65 & \\
\hline & Ex-fumante & 115 & 75.7 & 37 & 24.3 & 152 & \\
\hline Tabagismo & Nunca fumou & 91 & 74.0 & 32 & 26.0 & 123 & 0.9360 \\
\hline & Total & 254 & & 86 & & 340 & \\
\hline & Sim & 71 & 79.8 & 18 & 20.2 & 89 & \\
\hline $\begin{array}{l}\text { Bebida } \\
\text { Alcoólica }\end{array}$ & Não & 183 & 72.9 & 68 & 27.1 & 251 & 0.2004 \\
\hline & Total & 254 & & 86 & & 340 & \\
\hline & Não & 188 & 75.2 & 62 & 24.8 & 250 & \\
\hline $\begin{array}{c}\text { Incapacidade } \\
\text { ABVD }\end{array}$ & Sim & 66 & 73.3 & 24 & 26.7 & 90 & 0.7268 \\
\hline & Total & 254 & & 86 & & 340 & \\
\hline & Sim & 224 & 75.9 & 71 & 24.1 & 295 & \\
\hline $\begin{array}{l}\text { Incapacidade } \\
\text { AIVD }\end{array}$ & Não & 30 & 66.7 & 15 & 33.3 & 45 & 0.1829 \\
\hline & Total & 254 & & 86 & & 340 & \\
\hline & Sim & 90 & 75.0 & 30 & 25.0 & 120 & \\
\hline Polifarmácia & Não & 164 & 74.6 & 56 & 25.5 & 220 & 0.9266 \\
\hline & Total & 254 & & 86 & & 340 & \\
\hline
\end{tabular}


Tabela 2 - Prevalência de hipertensão arterial, segundo o modelo logístico para probabilidade de hipertensão, de idosos usuários da UBS Vila Santa Catarina, município de São Paulo-SP, 2013.

\begin{tabular}{lcccrc} 
& Parâmetro & GL & Estimativa & $\begin{array}{c}\text { Estatística de } \\
\text { Wald }\end{array}$ & p-valor \\
\hline Intercepto & & 1 & 0.9967 & 12.1415 & $\mathbf{0 , 0 0 0 5}$ \\
\hline \multirow{2}{*}{ Faixa idade } & 1 & 1 & 0.3359 & 1.4648 & 0.2262 \\
& 2 & 1 & -0.015 & 0.0037 & 0.9516 \\
& 3 & 1 & -0.3202 & 1.1345 & 0.2868 \\
\hline Sexo & 4 & 1 & 0.0936 & 0.0796 & 0.7778 \\
\hline Raça/cor & 1 & 1 & 0.376 & 4.6408 & $\mathbf{0 , 0 3 1 2}$ \\
\hline & 1 & 1 & -0.3329 & 2.8636 & 0.0906 \\
Estado civil & 2 & 1 & 0.5714 & 5.8427 & $\mathbf{0 , 0 1 5 6}$ \\
\hline Escolaridade & 1 & 1 & 0.3137 & 1.5424 & 0.2143 \\
\hline Renda familiar & 2 & 1 & -0.2998 & 1.7104 & 0.1909 \\
\hline Internação hospitalar & 3 & 1 & -0.176 & 0.3326 & 0.5641 \\
\hline Atividade física & 1 & 1 & 0.1291 & 0.7108 & 0.3992 \\
\hline Tabagismo & 2 & 1 & -0.0947 & 0.3263 & 0.5678 \\
\hline Bebida alcoólica & 1 & 1 & -0.2016 & 1.6796 & 0.1950 \\
\hline Incapacidade ABVD & 1 & 1 & -0.0084 & 0.0025 & 0.9600 \\
\hline Incapacidade AIVD & 1 & 1 & -0.4832 & 2.8875 & 0.0893 \\
\hline Polifarmácia & 2 & 1 & 0.1423 & 0.5338 & 0.4650 \\
\hline
\end{tabular}

\section{DISCUSSÃo}

Neste estudo, observou-se alta prevalência de HAS $(74,7 \%)$ entre os idosos pesquisados. Outros estudos que investigaram a prevalência de HAS em idosos, apontaram resultados inferiores ${ }^{10,11}$. Estudos internacionais, realizados na Europa e no Canadá, apontaram prevalência de 30\% e $35 \%$ respectivamente ${ }^{12,13}$. Embora, não se possa considerar que as doenças crônicas auto referidas correspondam de fato à prevalência das mesmas, estas tem sido utilizada em estudos epidemiológicos como um indicador do estado de saúde, especialmente em pessoas idosas ${ }^{13}$.

Estudo realizado com idosos residentes na comunidade, em área residencial do município de São Paulo, e outro no município de Tubarão-SC identificou maior prevalência de HAS em mulheres idosas ${ }^{14,15}$. Nesse estudo a maior prevalência de hipertensão ocorreu entre idosos do sexo masculino.

Quanto a escolaridade, encontrou-se maior prevalência da HAS em idosos com menor escolaridade. Esses dados são compatíveis com a prevalência nacional e com pesquisas que detectaram um perfil educacional baixo nos idosos brasileiros ${ }^{15-17}$.

Neste estudo observaram-se desvantagens socioeconômicas dos idosos com renda familiar de 1 a 3 salários mínimos 264 (77,6\%). A renda familiar representa um fator determinante na situação de saúde do idoso, possivelmente nesta fase da vida exista uma necessidade maior de medicamentos, presença de doenças crônicas, alimentação diferenciada e outros custos que o processo de limitação física acarreta. Além disso, devido às diversas mudanças ocorridas nos arranjos familiares nos últimos tempos, o idoso pode se deparar com uma realidade na qual se vê obrigado a amparar familiares desempregados ou doentes. Nesse contexto, cresce o número de estudos que mostram a relevância da figura do idoso aposentado como provedor da família18,19.

Quanto a internação hospitalar, esta variável não apresentou significância estatística, porém, o cálculo de razão de chance mostrou que os idosos hipertensos deste estudo apresentam de 2 a 3 vezes 
maiores chances de internação hospitalar. Embora em determinadas circunstâncias, a hospitalização seja a única possibilidade para o tratamento do idoso, ela tem como repercussões a diminuição da capacidade funcional, a recuperação mais lenta e prolongada, a demanda de tecnologias de alto custo - aumentando os gastos com assistência médica e, a necessidade de recursos humanos capacitados $^{20}$.

A inatividade física possivelmente está associada as alterações morfofuncionais inerentes ao processo de envelhecimento humano, quando estão associadas a doenças crônicas, podem levar à diminuição da independência física do idoso ${ }^{21}$. Estudo realizado na cidade de Ribeirão Preto/SP e que avaliou o nível habitual de atividade física em idosos, obteve associação positiva quanto à saúde cardiovascular de idosos hipertensos ${ }^{22}$.

No mundo existem aproximadamente $22 \%$ de homens com 60 anos ou mais que são fumantes e 8\% das mulheres têm esse hábito ${ }^{23}$. No Brasil, Inquérito de Saúde realizado no estado de São Paulo, em uma amostra de 1954 idosos, demonstrou alta prevalência de tabagismo em idosos do sexo masculino com baixa renda, menos anos de estudo, baixo peso corporal e inatividade física $^{23}$. No presente estudo, idosos tabagistas apresentaram de duas a três vezes mais chances de desenvolverem hipertensão em decorrência deste hábito.

A perda da capacidade funcional está associada à predição de fragilidade, dependência, institucionalização, risco aumentado de quedas e morte. De outro modo, problemas de mobilidade trazem complicações ao longo do tempo, gerando cuidados de longa permanência e alto custo em virtude da necessidade de assistência médica e risco de hospitalização, contribuindo significativamente para a atual crise no sistema de saúde ${ }^{24}$. Um estudo que avaliou 999 pessoas com média de idade de 68,5 anos demonstrou que pacientes com hipertensão tiveram um risco aumentado de desenvolver incapacidade funcional nas funções avaliadas em relação aos normotensos ${ }^{25}$.
Outro estudo realizado no município de São Paulo, com 1769 pessoas idosas classificaram os participantes de pesquisa como independentes ou dependentes para atividades básicas da vida diária e atividades instrumentais da vida diária. A hipertensão arterial foi a condição crônica mais frequente $(53,4 \% \text { da amostra })^{26}$. No estudo aqui apresentado, a prevalência de incapacidade para as AIVDs foi maior do que para as ABVDs. Embora não tenha havido significância estatística para essas variáveis, a razão de chance foi de uma a duas vezes. Indicando que idosos hipertensos apresentam baixa probabilidade de terem incapacidade funcionais.

0 uso de 5 ou mais medicamentos foi relatado por 35,2\% dos idosos. Esse resultado pode ter várias explicações. Em certa medida é uma consequência da maior prevalência de DCNT nesse grupo etário. Contribui, também, para a utilização de múltiplos medicamentos, a forma desarticulada como é feita a assistência à saúde do idoso, atendido em momentos próximos por diferentes especialistas, sem que o paciente seja questionado sobre quais medicamentos utiliza. Além disso, as receitas muitas vezes são repetidas indefinidamente porque os pacientes não são orientados acerca da duração do tratamento ${ }^{27}$.

A prevalência de polifarmácia foi maior do que a verificada em outros estudos brasileiros de base populacional com idosos, que também consideraram como polifarmácia o uso de 5 ou mais medicamentos. Nestes, a prevalência variou de $11,3 \%$ na região metropolitana de Belém-PA ${ }^{28}$ a 29,0\% na Bahia $^{29}$ e 32,7\% entre aposentados do Rio de Janeiro $^{30}$. As diferenças podem estar relacionadas às características das populações pesquisadas e a metodologia utilizada nos diferentes inquéritos.

0 estudo em questão possui algumas limitações que devem ser consideradas na interpretação dos resultados. Primeiro, tratase de um estudo transversal, ou seja, apesar de encontrar fatores relacionados à hipertensão arterial em idosos, não demonstra uma relação de causa-efeito. Por outro lado, apesar do estudo ter sido realizado com idosos 
vivendo numa comunidade, tais resultados podem não refletir a realidade em algumas regiões do município de São Paulo, podendo haver diferenças em outras.

\section{CONCLUSÃo}

Este estudo representa uma contribuição para o conhecimento dos fatores associados à hipertensão em idosos acompanhados eu um serviço de atenção primária. A prevalência da hipertensão arterial em idosos foi alta, quando comparada a outros estudos realizados no Brasil.

Os fatores associados à hipertensão foram: o gênero masculino e raça negra. Os idosos eram em sua maioria mulheres na faixa etária de 60 a 69 anos, baixa escolaridade, renda familiar insuficiente, dependentes do Sistema Único de Saúde e com alto grau de incapacidade para atividades instrumentais da vida diária.

0 aumento da população idosa, evidencia maior número de problemas crônicos, entre elas a hipertensão arterial, de alta prevalência nesta faixa da população. Estudos nesta área do conhecimento são importantes para o planejamento e o estabelecimento de políticas públicas em benefício da população idosa.

\section{REFERÊNCIAS}

1. Cruz DT, Ribeiro LC, Vieira MT, Teixeira MTB, Bastos RR, Leite ICB. Prevalência de quedas e fatores associados em idosos. Rev Saúde Pública. [Internet]. 2012 [citado em: 20 abr 2017]; 46(1):138-46. Disponível em: http://www.scielo.br/pdf/rsp/v46n1/3070.pdf http://dx.doi.org/10.1590/S0034-

89102011005000087

2. Ayama S, Feriancic MM. Fundamentos de gerontologia. In: Mendes TAC, Waksman RD, Farah OGD. Manuais de especialização: geriatria e gerontologia. São Paulo: Manole; 2014.

3. Veras RP. Prevenção de doenças em idosos: os equívocos dos atuais modelos. Cad Saúde Pública. [Internet]. 2012[citado em: 20 abr 2017]; 28(10):1834-40. Disponível em: http://www.scielo.br/pdf/csp/v28n10/03.pdf 4. Santos MV, Oliveira DC, Arraes LB, Oliveira DA, Medeiros L, Novaes MA. Adesão ao tratamento anti-hipertensivo: conceitos, aferição e estratégias inovadoras de abordagem. Rev Bras Clin Med. [Internet]. 2013 [citado em: 20 abr 2017];
11(1):55-61. Disponível

em: http://files.bvs.br/upload/S/1679-

1010/2013/v11n1/a3390.pdf

5. Freitas JGA, Nielson SEO, Porto CC. Adesão ao tratamento farmacológico em idosos hipertensos: uma revisão integrativa da literatura. Rev Soc Bras Clin Med. [Internet] 2015 [citado em: $30 \mathrm{abr}$ 2017]; 13(1):75-84. Disponível em: http://files.bvs.br/upload/S/1679-

1010/2015/v13n1/a4782.pdf

6. Santos JC, Florêncio RS, Oliveira CJ, Moreira TMM. Adesão do idoso para o tratamento da hipertensão arterial e intervenções de enfermagem. Rev RENE. [Internet]. 2012 [citado em: 05 maio 2017]; 13(2):343-53. Disponível em: http://www.periodicos.ufc.br/rene/article/view /3924/3114

7. Santos GS, Cunha ICKO. Avaliação da capacidade funcional de idosos para o desempenho das atividades instrumentais da vida diária: um estudo na atenção básica à saúde. Rev Enferm Cent-Oeste Min. [Internet]. 2013 [citado em: 20 fev 2017]; 3(3):820-8. Disponível em: http://www.seer.ufsj.edu.br/index.php/recom/a rticle/view/421/528

8. Fundação Sistema Estadual de Análise de Dados. Cidade de São Paulo registra rápido envelhecimento e reduz o ritmo de crescimento da população [Internet]. São Paulo: SEADE; 2015 [citado em 20 jan 2017]. Disponível em: http://www.seade.gov.br/cidade-de-sao-pauloregistra-rapido-envelhecimento-e-reduz-o-ritmode-crescimento-da-populacao

9. Johnston B, Reuben DB. Avaliação geriátrica. In: Williams BA, Chang A, Ahalt C, organizadores. Atualização, diagnóstico e tratamento em geriatria. Porto Alegre: Artmed; 2015. p. 24-28.

10. Mendes GS, Moraes CF, Gomes L. Prevalência de hipertensão arterial sistêmica em idosos no Brasil entre 2006 e 2010. Rev Bras Med Fam Comunidade. [Internet]. 2014 [Citado em: $10 \mathrm{mar}$ 2017]; 9(32):273-8. Disponível em: https://rbmfc.org.br/rbmfc/article/view/795/6 41. DOI: $10.5712 / \mathrm{rbmfc} 9$ (32)795

11. Pimenta FB, Pinho L, Silveira MF, Botelho ACC. Fatores associados a doenças crônicas em idosos atendidos pela Estratégia de Saúde da Família. Ciênc Saúde Coletiva. [Internet]. 2015 [citado em: 5 abr 2017]; 20(8):2489-98. Disponível em: http://www.scielo.br/pdf/csc/v20n8/1413-

8123-csc-20-08-2489.pdf

DOI: http://dx.doi.org/10.1590/1413-

81232015208.11742014

12. Allen M, Kelly K, Fleming I. Hypertension in elderly patients recommended systolic targets are not evidence based. Can Fam Physician. [Internet] 
2013 [Acesso em: 15 jun 2017]; 59(1):19-21. Disponível em: https://www.ncbi.nlm.nih.gov/pmc/articles/PM C3555642/pdf/0590019.pdf

13. Olives C, Myerson R, Mokdad AH, Murray CJL, Lim SS. Prevalence, awareness, treatment, and control of hypertension in United States countries, 2001-2009. PloS ONE [Internet] 2013 [acesso em: 10 abr 2017]; 8(4):1-8. Disponível em: https://www.ncbi.nlm.nih.gov/pmc/articles/PM C3618269/pdf/pone.0060308.pdf

14. Orsi E, Xavier AJ, Ramos LR. Trabalho, suporte social e lazer protegem idosos da perda funcional: estudo epidoso. Rev Saúde Pública. [Internet] 2011[acesso em: 10 abr 2017]; 45(4);685-92. Disponível em: http://www.scielo.br/pdf/rsp/v45n4/2626.pdf. Doi: $\quad$ http://dx.doi.org/10.1590/S003489102011000400007

15. Nunes TM, Martins AM, Manoel Al, Trevisol DJ, Schuelter-Trevisol F, Cavalcante RASQ et al. Hypertension in Elderly Individuals from a City of Santa Catarina: A Population-Based Study. Int J CardiovascSci. [Internet] 2015 [acesso em: $10 \mathrm{fev}$ 2017]; 8(5):370-6. Disponível em: http://www.onlineijcs.org/sumario/28/pdf/en_v 28n5a05.pdf. DOI: 10.5935/23594802.20150055

16. VI Diretrizes Brasileira de Hipertensão. Arq Bras Cardiol. [Internet] 2010 [acesso em: $10 \mathrm{mar}$ 2017]; 95(1 Supl1):1-64. Disponível em: http://www.scielo.br/pdf/abc/v95n1s1/v95n1s 1.pdf. http://dx.doi.org/10.1590/S0066782X2010001700001

18. Santos MIPO, Griep RH. Capacidade funcional de idosos atendidos em um programa do SUS em Belém (PA). Ciênc Saúde Coletiva. [Internet] 2013 [acesso em: 5 fev 2017]; 18(3):753-61. Disponível em: http://www.scielo.br/pdf/csc/v18n3/21.pdf. Doi: http://dx.doi.org/10.1590/S141381232013000300021

19. Bento JA, Lebrão ML. Suficiência de renda percebida por pessoas idosas no Município de São Paulo/Brasil. Ciênc Saúde Coletiva. [internet] 2013 [acesso em: 15 abr 2017]; 18(8):2229-38. Disponível http://www.scielo.br/pdf/csc/v18n8/07.pdf http://dx.doi.org/10.1590/S1413-

81232013000800007

20. Pagotto V, Silveira EA, Velasco WD. Perfil das hospitalizações e fatores associados em idosos usuários do SUS. Ciênc Saúde Coletiva. [internet] 2013 [acesso em: 10 jun 2017]; 18(10):3061-70. Disponível em: http://www.scielo.br/pdf/csc/v18n10/v18n10a 31.pdf. http://dx.doi.org/10.1590/S141381232013001000031

21. Santos SSC. Concepções teórico-filosófica sobre envelhecimento, velhice, idoso e enfermagem geronto-geriátrica. Rev Bras Enferm. [Internet] 2010 [acesso em: 10 jul 2017]; 63(6):1035-9. Disponível em: http://www.scielo.br/pdf/reben/v63n6/25.pdf. http://dx.doi.org/10.1590/S0034-

71672010000600025

22. Trapé AA, Lizzi EASS, Jacomini AM, Bueno JCR, Zago AS. Aptidão física e nível habitual de atividade física associados à saúde cardiovascular de adultos e idosos. RevBrasMed Esporte. [Internet] 2015 [acesso em: 10 jul 2017]; 48(5):457-66. Disponível em: http://www.revistas.usp.br/rmrp/article/view/ 112592/110493 http://dx.doi.org/10.11606/issn.2176-

7262.v48i5p457-466

23. Zaitune MPA, Barros MBA, Lima MG, César CLG, Carandina L, Goldbaum M, et al. Fatores associados ao tabagismo em idosos: inquérito de saúde no estado de São Paulo (ISA-SP). Cad Saúde Pública. [Internet] 2012 [acesso em: 5 jul. 2017]; 28(3):583-95. Disponível em: http://www.scielo.br/pdf/csp/v28n3/18.pdf http://dx.doi.org/10.1590/S0102-

311X2012000300018

24. Lucio A, Bezerra MJC, Sousa SA, Miranda MLJ. Características da capacidade funcional e sua relação com o IMC em idosas ingressantes em um programa de educação física. RevBrasCiênc Mov. [Internet] 2011[acesso em: 20 jul 2017]; 19(2):13-8. Disponível em: https://portalrevistas.ucb.br/index.php/RBCM/a rticle/view/2415/1894

25. Hajjar I, Lackland D, Cupples LA, Lipsitz LA. The association between concurrent and remote blood pressure and disability in older adults. Hypertension. [Internet] 2007 [acesso em: $20 \mathrm{fev}$ 2017]; 50(6):1026-32. Disponível em: https://www.ncbi.nlm.nih.gov/pmc/articles/PM C2424316/pdf/nihms43061.pdf

26. Alves LC, Leimann BCQ, Vasconcelos MEL, Carvalho MS, Vasconcelos AGG, Fonseca TCO, et al. A influência das doenças crônicas na capacidade funcional dos idosos do Município de São Paulo, Brasil. Cad Saúde Pública. [Internet] 2007 [acesso em: 10 abr 2017]; 23(8):1924-30. Disponível em: http://www.scielo.br/pdf/csp/v23n8/19.pdf. http://dx.doi.org/10.1590/S0102-

311X2007000800019

27. Carvalho MFC, Lieber NSR, Mendes GB, Secoli SR, Ribeiro E, Lebrão ML, et al. Polifarmácia entre idosos do Município de São Paulo - Estudo SABE. 


\section{Santos GS, Cunha ICKO}

Rev Bras Epidemiol. [Internet] 2012 [acesso em: 20 jul 2017]; 15(4):817-27. Disponível em: http://www.scielo.br/pdf/rbepid/v15n4/13.pdf http://dx.doi.org/10.1590/S1415-

790X2012000400013

28. Cuentro VS, Modesto T, Andrade MA, Silva MVS. Prevalência e fatores associados à polifarmácia entre idosos de um hospital público. Rev Contexto Saúde. [Internet] 2016 [acesso em: 10 fev 2017]; 16(30):28-35. Disponível em: https://www.revistas.unijui.edu.br/index.php/co ntextoesaude/article/view/4448/4981. DOI: http://dx.doi.org/10.21527/2176-

7114.2016.30.28-35

29. Sales AS, Sales MGS, Casotti CA. Perfil farmacoterapêutico e fatores associados à polifarmácia entre idosos de Aiquara, Bahia, em 2014. EpidemiolServ Saúde. [Internet]. 2017 [acesso em: 14 fev 2017]; 26(1): 121-32. Disponível

\section{Saúde do Idoso}

http://www.scielo.br/scielo.php?script=sci_artte xt\&pid=S2237-96222017000100121\&lng=pt.

Doi: $\quad$ http://dx.doi.org/10.5123/s167949742017000100013

30. Rozenfeld S, Fonseca MJM, Acurcio FA. Drug utilization and polypharmacy among the elderly: a survey in Rio de Janeiro City, Brazil. Rev. Panam. Salud Pública. [Internet]. 2008 [acesso em: 22 jul 2017]; 23:34-43. Disponível em: https://scielosp.org/scielo.php?script=sci_arttext \&pid=S1020-

49892008000100005\&lng=en\&nrm=iso\&tlng=en .http://dx.doi.org/10.1590/S102049892008000100005

\section{CONTRIBUIÇÕES}

Todos os autores tiveram iguais contribuições no desenho do estudo, análise e redação final.

\footnotetext{
Como citar este artigo (Vancouver)

Santos GS, Cunha ICKO. Prevalência e fatores associados à hipertensão em idosos de um serviço de atenção primária. REFACS [Internet]. 2018 [citado em inserir dia, mês e ano de acesso]; 6(Supl. 1): 321-329. Disponível em: inserir link de acesso. DOI: inserir link do DOI.

Como citar este artigo (ABNT)

SANTOS, G. S.; CUNHA, I. C. K. O. Prevalência e fatores associados à hipertensão em idosos de um serviço de atenção primária. REFACS, Uberaba, v. 6, p. 321-329, 2018. Supl. 1. Disponível em: <inserir link de acesso>. Acesso em: inserir dia, mês e ano de acesso. DOI: inserir link do DOI.

\section{Como citar este artigo (APA)}

Santos, G. S. \& Cunha, I. C. K. O. (2018). Prevalência e fatores associados à hipertensão em idosos de um serviço de atenção primária. REFACS, 6(Supl. 1), 321-329. Recuperado em: inserir dia, mês e ano de acesso e, inserir link de acesso. DOI: inserir link do DOI.
} 